\title{
Effect of Ginkgo Biloba Extract on N-Methyl-D-Aspartic Acid Receptor Subunit 2B Expression in a Salicylate-Induced Ototoxicity Model
}

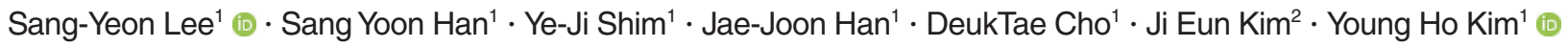 \\ Departments of ${ }^{1}$ Otorhinolaryngology-Head and Neck Surgery and ${ }^{2}$ Pathology, SMG-SNU Boramae Medical Center, \\ Seoul National University College of Medicine, Seoul, Korea
}

Objectives. Sodium salicylate (SS) is well known for its ototoxic properties that induce functional and morphological changes in the cochlea and brain. Ginkgo biloba extract (GBE) has been widely used for treatment of various neurodegenerative diseases; however, its effects on salicylate-induced ototoxicity remain unclear. Herein, we examined the effects of EGb 761 (EGb), a standard form of GBE, on the plasticity of the N-methyl-D-aspartate receptor subunit 2B (GluN2B) in the inferior colliculus (IC) following SS administration.

Methods. Seven-week-old Sprague Dawley rats $(n=24)$ were randomly allocated to control, SS, EGb, and EGb+SS groups. The SS group received a single intraperitoneal SS injection $(350 \mathrm{mg} / \mathrm{kg})$, the EGb group received EGb orally for 5 consecutive days $(40 \mathrm{mg} / \mathrm{kg})$, and the $\mathrm{EGb}+\mathrm{SS}$ group received $\mathrm{EGb}$ for 5 consecutive days, followed by an SS injection. The auditory brainstem responses (ABRs) were assessed at baseline and 2 hours after SS administration. GluN2B expression was examined by Western blot and immunohistochemistry.

Results. There were no significant differences in ABR threshold shifts among the groups. The expression of the GluN2B protein normalized by which of glyceraldehyde 3-phosphate dehydrogenase (GAPDH) was significantly lower in the $\mathrm{EGb}+\mathrm{SS}$ group, as compared to the SS group $(P=0.012)$. Weak and diffused GluN2B immunoreactivity was detected in the IC neural cells of the EGb+SS group, while those of the SS group exhibited strong and diffused GluN2B positivity.

Conclusion. EGb may play a role in regulating the GluN2B expression in the IC of salicylate-induced ototoxicity model.

Keywords. N-Methyl-D-Aspartate Receptor Subunit 2B; Ginkgo Biloba Extract; Inferior Colliculus

\section{INTRODUCTION}

Salicylate is an active component of aspirin with well-established ototoxic properties [1]. Salicylate, especially in large doses $(250-300 \mathrm{mg} / \mathrm{kg})$, is known to interfere with outer hair cells $(\mathrm{OHC})$ electromotility in animal models, by displacing chloride

\footnotetext{
- Received June 26, 2018

Revised August 18, 2018

Accepted September 14, 2018

- Corresponding author: Young Ho Kim

Department of Otorhinolaryngology-Head and Neck Surgery, SMG-SNU Boramae Medical Center, Seoul National University College of Medicine, 20 Boramae-ro 5-gil, Dongjak-gu, Seoul 07061, Korea

Tel: +82-2-870-2442, Fax: +82-2-870-3863

E-mail: yhkiment@gmail.com
}

and binding to the anion-binding sites on prestin. This in turn suppresses the amplification properties of the cochlea and elevates the hearing threshold [2].

$\mathrm{N}$-methyl-D-aspartate receptor (NMDA-R) is a heteromeric complex (GluN1, GluN2, GluN3), which gets activated upon glycine and glutamate binding and is involved in neuronal excitotoxicity [3]. NMDA-R functions as an important neuromodulator in the cochlear hair cell synapse [4]. Salicylate administration is known to induce glutamate-mediated excitotoxicity through the upregulation of NMDA-R in ascending/descending central/peripheral auditory pathways $[5,6]$. Among four different GluN2 isoforms, NMDA-R subunit 2B (GluN2B) appears to be significantly elevated in the inferior colliculus (IC) and auditory cortex (AC) in response to salicylate in rodents [7]. Considering

Copyright @ 2019 by Korean Society of Otorhinolaryngology-Head and Neck Surgery.

This is an open-access article distributed under the terms of the Creative Commons Attribution Non-Commercial License (http://creativecommons.org/licenses/by-nc/4.0)

which permits unrestricted non-commercial use, distribution, and reproduction in any medium, provided the original work is properly cited. 


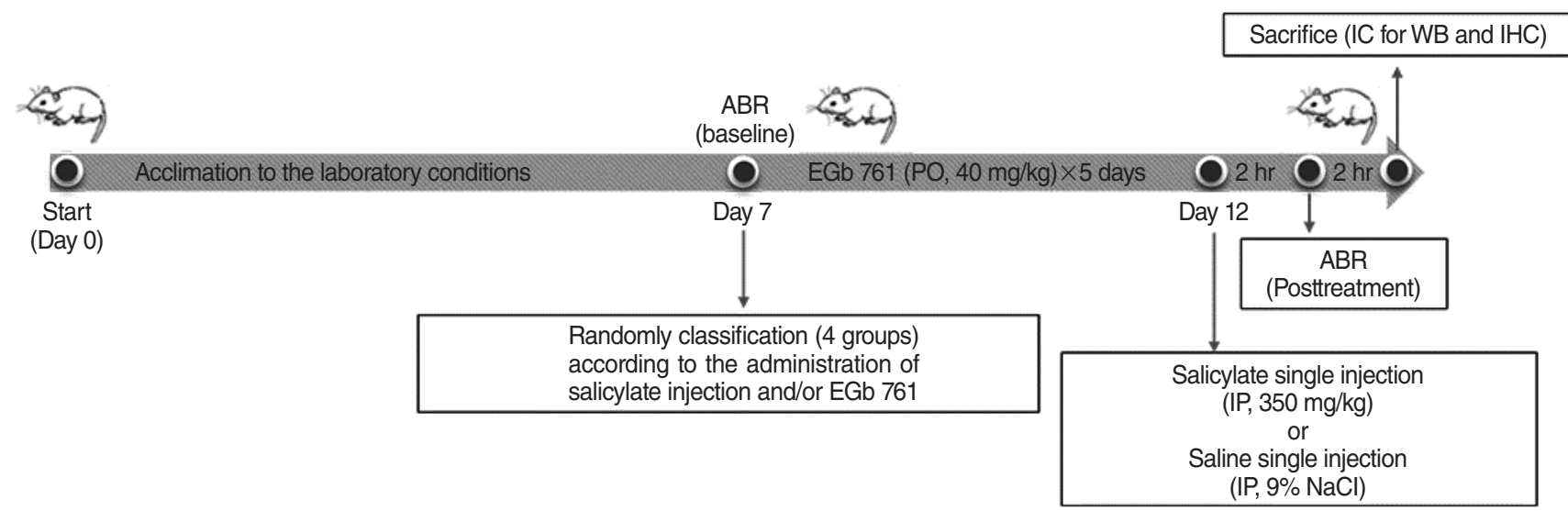

Fig. 1. Schematic of the study experimental protocol using a salicylate-induced ototoxicity rat model. ABR, auditory brainstem response; PO, orally; IC, inferior colliculus; WB, Western blot; IHC, immunohistochemistry; IP, intraperitoneally.

that the GluN2B subunit mediates most of the NMDA current and that activation of GluN2B-containing NMDA-Rs results in excitotoxicity and neuronal apoptosis [8,9], GluN2B pathophysiology might mediate salicylate-induced excitotoxicity.

Natural products with neuroprotective properties have been widely acknowledged [10], among which, the therapeutic effects of Ginkgo biloba extract (GBE) on various neurodegenerative diseases. More specifically, GBE has been reported to exert beneficial effects on NMDA-R-associated excitotoxicity in isolated rat hippocampal neurons [11]. GBE is also shown to prevent excitotoxic damage in mouse cochlea isolations, by moderately inhibiting $\mathrm{Na}^{+}$channels at depolarized potential through the blockade of GluN2B [8].

Taking into consideration the aforementioned studies, EGb 761 (EGb), a standard form of GBE, is likely to play a role in GluN2B regulation during salicylate-induced ototoxicity. Given that the plasticity of GluN2B expression after salicylate administration along with auditory pathway, therefore, we hypothesize the changes of GluN2B depending on EGb pretreatment will be observed in auditory center. To date, the effects of EGb on IC neurophysiology during salicylate-induced ototoxicity have nev-

\section{HIIGHLIIGHTS}

- Sodium salicylate (SS)-induced ototoxicity elicits increased expression of N-methyl-D-aspartate receptor subunit 2B (GluN2B) in the inferior colliculus (IC).

- EGb 761, a standard form of Ginkgo biloba extract, decreases the GluN2B protein expression in the IC of SS-induced ototoxicity model based on Western blot.

- EGb 761 decreases the expression of immunoreactive GluN2B in the IC of SS-induced ototoxicity model based on immunohistochemistry.

- EGb 761 may play a role in regulating the GluN2B expression in the IC of the rat SS-induced ototoxicity model. er been evaluated. Herein, we examine the effects of EGb on GluN2B-mediated plasticity in central auditory regions immediately after sodium salicylate (SS) administration, in a rat salicylate-induced ototoxicity model.

\section{MATERIALS AND METHODS}

\section{Animal care}

Seven-week-old male Sprague Dawley rats (weight, 180-220 g) were acclimatized under specific pathogen free conditions with a 12-hour light/dark cycle for 1 week. Twenty-four rats were randomly allocated to four groups: control, SS, EGb 761 (EGb), or EGb+SS.The SS group received a single salicylate $(350 \mathrm{mg} / \mathrm{kg}$, intraperitoneally, Cat \#S3007; Sigma-Aldrich, St. Louis, MO, USA) and the EGb group was treated with EGb $(40 \mathrm{mg} / \mathrm{kg}$, orally, Ginexin; SK Chemical, Seoul, Korea) for 5 consecutive days before they were sacrificed and the $\mathrm{EGb}+\mathrm{SS}$ group received $\mathrm{EGb}$ orally for 5 consecutive days, followed by SS intraperitoneally injection 2 hours after the final EGb administration. All experimental timelines are summarized in Fig. 1.

All animal care and experimental procedures were approved by the Institutional Animal Care and Use Committee Health System (IACUC No. 2017-0027) of Seoul National University Hospital, Korea and were conducted in accordance to the internationally accepted Principles for Laboratory Animal Use and Care, as stated in the U.S. guidelines [12].

\section{Auditory brainstem response}

Left ear hearing in all animals was estimated by auditory brainstem responses (ABRs) at baseline and 2 hours after SS administration. Prior to ABR assessment, animals were anesthetized by intramuscular injection of Zoletil $(40 \mathrm{mg} / \mathrm{kg}$ ) and xylazine $(10 \mathrm{mg} / \mathrm{kg})$. ABR assessment was performed in an acoustically insulated booth. Platinum needle electrodes were placed subcutaneously on the vertex (positive), ipsilateral (negative), and 
contralateral (ground) ear. The Intelligent Hearing Systems (IHS; Miami, FL, USA), employing IHS high-frequency transducers (HFT9911-20-0035) and IHS high-frequency software (ver. 3.30), were used to measure ABRs. Three tone-burst sounds of 8 , 16 , and $32 \mathrm{kHz}$ were applied as sound stimuli (duration, 1,562 $\mu \mathrm{m}$; CoS shaping, $21 \mathrm{~Hz}$ ). Before the electroencephalography signal acquisition, the impedance between the electrodes was monitored so as not to exceed $2 \mathrm{k} \Omega$. The responses were amplified $(\times 100,000)$ and band pass-filtered $(100-1,500 \mathrm{~Hz})$. Then, the responses at each intensity level were averaged for 512 sweeps. The intensity level of the stimuli changed from $90 \mathrm{~dB}$ sound pressure level (SPL) to $10 \mathrm{~dB}$ SPL, by $5 \mathrm{~dB}$ SPL. ABR recordings were interpreted by an audiologist, who received no information regarding the experiments. The lowest intensity level of the stimuli that showed a respectable waveform in the ABR trace was determined as the hearing level.To analyze the changes in hearing levels, we calculated the threshold shift by subtracting the baseline threshold from each posttreatment threshold, accordingly. According to this method, a positive threshold shift represents a hearing level deterioration. After the ABR assessment, animals were sacrificed with deep anesthesia and their brains were rapidly harvested for Western blotting and immunohistochemistry.

\section{Western blotting}

Extracted brains were washed in ice-cold phosphate buffered saline (PBS) twice, homogenized and frozen in liquid nitrogen. The homogenized samples were lysed with radioimmunoprecipitation assay (RIPA) buffer (9806; Cell Signaling Technology, Danvers, MA, USA) containing protease/phosphatase inhibitor cocktails (5872, Cell Signaling Technology). Protein concentration was determined with the Bradford assay, and equal amounts of protein $(30 \mu \mathrm{g})$ were resolved in Mini-PROTEAN TGX Precast Gels (Bio-Rad, Hercules, CA, USA). The samples were then transferred to a polyvinylidene fluoride membrane. Primary antibodies for GluN2B and glyceraldehyde 3-phosphate dehydrogenase (GAPDH) were incubated overnight at $4^{\circ} \mathrm{C}$, while the horseradish peroxidase-conjugated secondary antibody was incubated at room temperature (RT) for 1 hour. The antibodies used in this study were as follows: 1:1,000 rabbit anti-GluN2B antibody (4212S, Cell Signaling Technology) and 1:5,000 goat anti-rabbit IgG horseradish peroxidase (Jackson ImmunoResearch, West Grove, PA, USA). Chemiluminescent substrate was applied on the membrane for 1 minute before film development. The intensities of the protein bands on the developed films were quantitated by Amersham Image 600 (GE Healthcare, Uppsala, Sweden) and analyzed by ImageQuant TL (GE Healthcare).

\section{Immunohistochemistry}

Dissected IC were fixed with $4 \%$ paraformaldehyde in $0.1 \mathrm{M}$ PBS overnight at $4^{\circ} \mathrm{C}$ and were then rinsed in $0.1 \mathrm{M}$ PBS con- taining 10\% sucrose for 1 hour. The samples were then decalcified with Calci-Clear Rapid (National Diagnostics, Atlanta, GA, USA) for 6 hours at RT and washed with PBS. Finally, the samples were sequentially rinsed in PBS with $10 \%$ sucrose, $20 \%$ sucrose, or $30 \%$ sucrose for 1 hour at RT, for each washing step. For immunohistochemistry, the sections were incubated in blocking solution (PBS containing $0.1 \%$ Tween 20 and $2 \%$ bovine serum albumin) for 60 minutes at RT, and then incubated with the rabbit anti-GluN2B primary antibody diluted in the blocking solution, overnight at $4^{\circ} \mathrm{C}$. Following that, streptavidinperoxidase was incubated with the sections at RT for $30 \mathrm{~min}$ utes. Finally, 3,3'-diaminobenzidine was applied for visualization. Sections were counterstained with hematoxylin and imaged under a light microscope. An experienced pathologist blindly assigned a pathology grading to all immunohistochemistry sections based on neural cell intensity and frequency. Glial cell was excluded from our analysis. Immunohistochemical expression of GluN2B in the IC was classified according to a 3-tiered system depending on fluorescence intensity (negative, weak positive, strong positive).

\section{Statistical analysis}

All data were analyzed using IBM SPSS ver. 22.0 (IBM Corp., Armonk, NY, USA). ABR threshold shifts and Western blots were analyzed by means of repeated-measures analysis of variance. Post-hoc testing was performed with the Tukey multiple comparison test, assessing the mean difference, standard error, and $95 \%$ confidence interval. The $P$-values $<0.05$ were considered to indicate statistical significance.

\section{RESULTS}

\section{ABR threshold shifts}

The average baseline and post-experimental left ear ABR

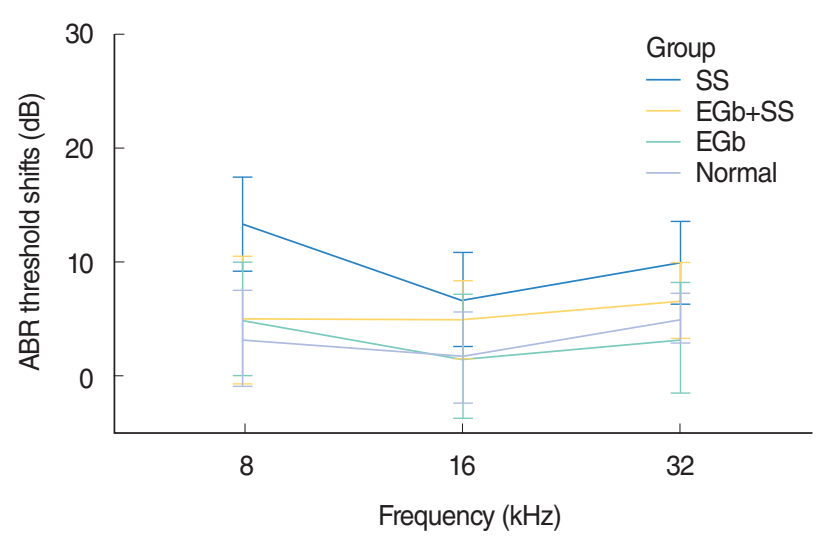

Fig. 2. Auditory brainstem responses (ABRs). Comparison analysis showed no significant differences in the ABR threshold shifts among the groups. Error bars represent the standard error. SS, sodium salicylate; EGb, EGb 761 Ginkgo biloba extract. 
thresholds are presented in Fig. 2. At baseline, the average ABR thresholds among the groups were not significantly different. The average ABR thresholds at $8 \mathrm{kHz}$ after the termination of the treatment shifted in the control, SS, EGb, and EGb+SS groups by $3.3 \mathrm{~dB}(\mathrm{SD}, 4.2 \mathrm{~dB}), 13.3 \mathrm{~dB}(\mathrm{SD}, 4.2 \mathrm{~dB}), 5.0 \mathrm{~dB}$ $(\mathrm{SD}, 4.9 \mathrm{~dB})$, and $5.0 \mathrm{~dB}(\mathrm{SD}, 5.6 \mathrm{~dB})$, respectively. The equivalent threshold shifts at $16 \mathrm{kHz}$ were $1.6 \mathrm{~dB}(\mathrm{SD}, 4.0 \mathrm{~dB}), 6.7 \mathrm{~dB}$ $(\mathrm{SD}, 4.2 \mathrm{~dB}), 1.6 \mathrm{~dB}(\mathrm{SD}, 5.4 \mathrm{~dB})$, and $5.0 \mathrm{~dB}(\mathrm{SD}, 3.4 \mathrm{~dB})$, respectively, and those at $32 \mathrm{kHz}$ were $5.0 \mathrm{~dB}(\mathrm{SD}, 2.2 \mathrm{~dB}), 10$ $\mathrm{dB}(\mathrm{SD}, 3.7 \mathrm{~dB}), 3.3 \mathrm{~dB}(\mathrm{SD}, 4.9 \mathrm{~dB})$, and $6.7 \mathrm{~dB}(\mathrm{SD}, 3.3 \mathrm{~dB})$, respectively. In this study, rats receiving intraperitoneal injections of salicylate $(350 \mathrm{mg} / \mathrm{kg})$ demonstrated approximately a $10 \mathrm{~dB}$ threshold shift at 2 hours. Although there is a tendency of a higher threshold shift in SS group than that in control group $(8 \mathrm{kHz}, P=0.064 ; 32 \mathrm{kHz}, P=0.082)$, the threshold shifts at each frequency were not significantly different among the groups ( $8 \mathrm{kHz}, P=0.466 ; 16 \mathrm{kHz}, P=0.801 ; 32 \mathrm{kHz}, P=0.622$ ).

\section{GluN2B expression analysis by Western blotting}

IC for the Western blot was harvested from brains of three rats in each group. NMDA-R GluN2B subunit expression in the IC evaluated for each group by Western blotting is shown in Fig. 3 .

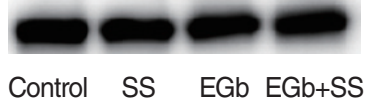

GAPDH expression in inferior colliculus

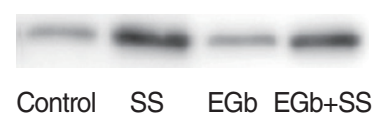

A GluN2B expression in inferior colliculus *

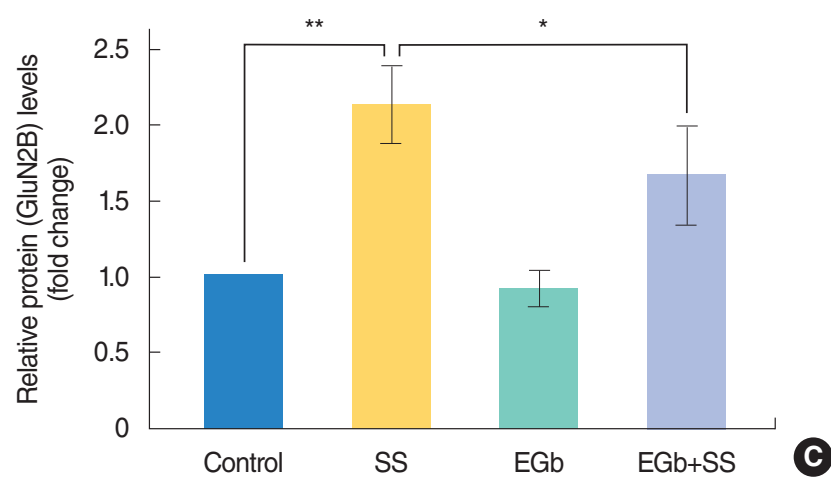

Fig. 3. Western blot analysis of the $\mathrm{N}$-methyl-D-aspartate receptor (NMDA-R) subunit 2B (GluN2B) in the inferior colliculus (IC). (A) The expression of glyceraldehyde 3-phosphate dehydrogenase (GAP$\mathrm{DH})$ protein among the four groups. (B) The expression of the GluN2B protein was significantly higher in the SS group compared to the control group. In addition, GluN2B protein expression was significantly lower in the EGb+SS group, as compared to the SS group. (C) Relative protein expression of GluN2B in the IC. The expression of GluN2B protein was significantly higher in the SS group and EGb+SS group compared to the control and EGb groups. Notably, the expression of GluN2B protein was significantly decreased in $\mathrm{EGb}+\mathrm{SS}$ group following EGb pretreatment compared to that in SS group. SS, sodium salicylate; EGb, EGb 761 Ginkgo biloba extract. ${ }^{\star} P<0.05$ (the SS group vs. EGb+SS group). ${ }^{* *} P<0.01$ (the control group vs. SS group).
Compared to the control and EGb groups, the expression of GluN2B in the SS and EGb+SS groups was significantly increased. Notably, the expression of GluN2B in the EGb+SS group showed a significantly decreased intensity than that in the SS group $(P=0.010)$.

\section{GluN2B expression analysis by immunohistochemistry}

Whole brains for the immunohistochemistry (IHC) analysis were harvested from three rats in each group. GluN2B expression could not be detected by immunohistochemistry in the IC of three rats allocated the control group (Fig. 4A). Similarly, all brain sections from the three rats treated with $\mathrm{EGb}$ alone showed no immunoreactivity against GluN2B (Fig. 4B). Brain sections from the three rats allocated the SS-treated group showed strong immunoreactivity against GluN2B in neuronal cells along with weak positivity in surrounding glial cells (Fig. 4C). This pattern characterized by strong activity of neuronal cells was observed in all of SS group. However, brain sections from the three rats treated with both $\mathrm{EGb}+\mathrm{SS}$ revealed a markedly reduced expression of GluN2B with weak positivity in neuronal cells. (Fig. 4D).
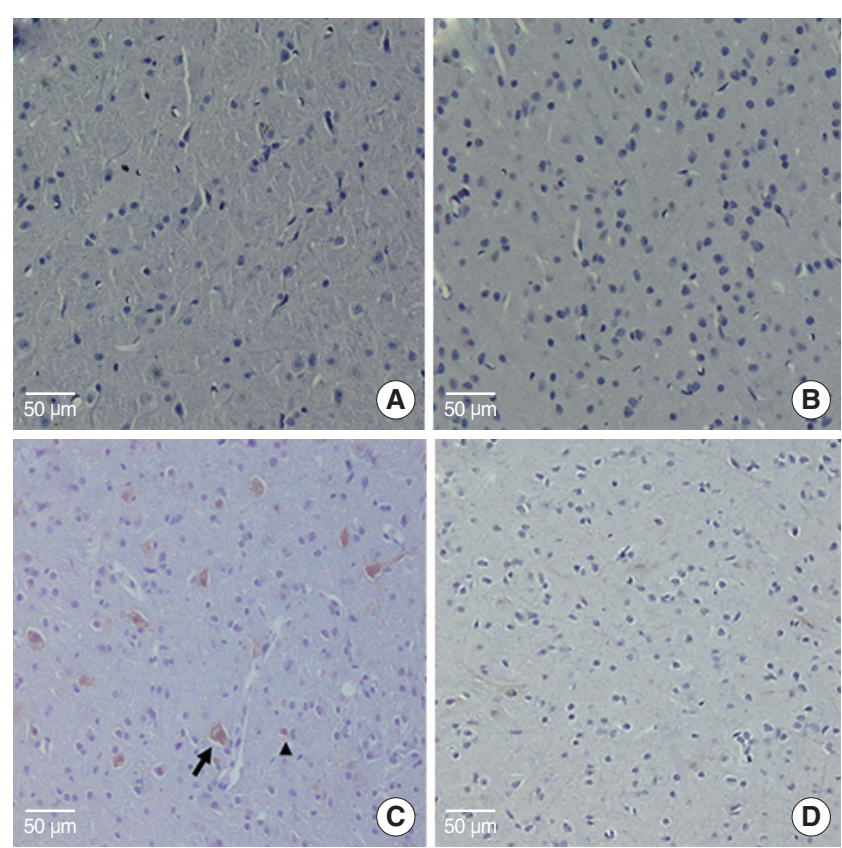

Fig. 4. The N-methyl-D-aspartate receptor (NMDA-R) subunit 2B (GluN2B) immunohistochemistry in the inferior colliculus $(\times 200$ magnification). (A) The control group showed no GluN2B immunoreactivity. (B) Brain samples from the EGb-treated group also showed negative GluN2B staining. (C) In the SS-treated group, strong immunoreactivity was detected in neuronal cells (arrow) along with some scattered glial cell immunoreactivity (arrowhead). (D) GluN2B staining vanished in the EGb+SS group, with only weak focal immunoreactivity. EGb, EGb 761 Ginkgo biloba extract; SS, sodium salicylate. 


\section{DISCUSSION}

The aim of this study was to investigate the therapeutic effects of EGb on brain excitotoxicity following acute in vivo salicylateinduced ototoxic damage. Salicylate administration is known to increase GluN2B expression in central auditory regions [7]. The present study showed that EGb attenuates SS-induced GluN2B upregulation in the IC. Considering these results, EGb may exert therapeutic effects on salicylate-induced ototoxicity by inhibiting the GluN2B NMDA-R subunit expression apart from excitotoxicity or neuronal apoptosis as suggested previously. However, the clinical and pathophysiological role of EGb on preventive GluN2B upregulation awaits further confirmation.

\section{NMDA-R expression in the salicylate-induced ototoxicity model} Salicylate's ototoxic properties have been well reported, demonstrating tinnitus and a sensorineural hearing loss when administered in high doses [13]. Although a significant salicylate-induced threshold shift was reported in some studies [13,14], the threshold shifting of ABR can be variable. A study employing the expression of insulin-like growth factors in a mouse model of salicylate ototoxicity exhibited approximately $30 \mathrm{~dB}$ ABR threshold shift [14], whereas the threshold shifting of ABR was less than $10 \mathrm{~dB}$ in a guinea pig model [15]. In this study, the threshold shifting of ABR was approximately $10 \mathrm{~dB}$, and there is a tendency of a higher threshold shift in SS group than that in control group. Additionally, in the previous study, salicylate did not change the amplitudes recorded from the IC when compared pre- and 2 hours post-systemic administration of SS, suggesting electrophysiological responses in the IC seem not show sound-evoked hyperactivity following salicylate administration unlike higher levels in the central auditory system [13]. The threshold shift is largely attributable to salicylate inhibitory effects on OHC electromotility. Previous studies showed that salicylate-induced hearing impairment is resulted from the impaired OHC sound amplification due to its direct action on $\mathrm{OHC}$ sensor and/or motor motility [2]. This phenomenon is known as salicylate-induced abolishment of otoacoustic emission (OAE) [2]. In addition, SS has been reported to increase spontaneous auditory nerve activity, then act on NMDA-Rs to enable responses to glutamate that is spontaneously released from inner hair cells [16]. Glutamate is the primary excitatory neurotransmitter in the central nervous system, but excess levels of it can precipitate neuronal dysfunction through the activation of NMDA-Rs and subsequent cellular excitotoxicity. In line with the present study, GluN2B expression in auditory-related brain areas increases after intraperitoneally SS injection $[7,17,18]$. GluN2B may play a role in mechanisms underlying excitotoxicity and subsequent neuronal apoptosis by regulating the peak amplitude of NMDA-R-mediated excitatory postsynaptic potentials. GluN2B-containing NMDA-Rs have been reported to increase neuronal excitability and neurotransmission in central auditory regions by increasing $\mathrm{Ca}^{2+}$ influx [19]. In support of our findings, other studies have also detected a GluN2B upregulation in response to SS administration in the IC $[7,18]$.

\section{Protective effects of EGb through GluN2B regulation}

The neuroprotective effect of EGb on excitotoxic neuronal damage has been consistently reported [11]. For example, GBE promoted the restoration capacity of hair cell functions by enhancing dopamine release and by moderately inhibiting $\mathrm{Na}^{+}$channels at depolarized potentials through GluN2B blockade in murine cochlea isolations [8]. Recently, SS was shown to elevate intracellular $\mathrm{Ca}^{2+}$ concentration and activate the $\mathrm{Ca}^{2+} / \mathrm{CaMKII} /$ CREB signaling cascade in AC, causing tinnitus in rats [20]. Conversely, EGb appeared to ameliorate glutamate-induced excitotoxicity and apoptosis by regulating intracellular $\mathrm{Ca}^{2+}$ concentration [21]. In line with these findings, we demonstrated that $\mathrm{EGb}$ pretreatment attenuated GluN2B upregulation in the rat IC immediately after SS administration. Considering that GluN2B plays a pivotal role in glutamate-induced excitotoxicity, its inhibition by EGb may attribute to calcium influx downregulation in the salicylate-induced ototoxicity model.

In addition, previous studies reported that proinflammatory cytokines might interact with NMDA-Rs or modulate NMDA-R gene expression [22]. Especially, tumor necrosis factor $\alpha$ (TNF- $\alpha$ ), interleukin (IL)-1 $\beta$, and GluN2B expression in the cochlea and IC were significantly increased in response to SS, inducing tinnitus, while cyclooxygenase-2 inhibition significantly reduced inflammatory responses and prevented neural damage in mice [23]. GBE has been recently shown to exert neuroprotective effects in response to neurotoxin-induced hippocampal injury in rats. This neuroprotection is mediated through a GBE-associated decrease in proinflammatory cytokines, such as TNF- $\alpha$, IL-1 $\alpha$, and IL-6, suggestive of the GBE anti-inflammatory properties [24]. Although the anti-inflammatory properties of EGb were not examined in the present study, it is likely that the GluN2B downregulation in the IC may be associated with such anti-inflammatory properties.

\section{Strengths and limitations of the current study}

To the best of our knowledge, this is the first study to examine how GluN2B expression levels in IC alter following SS treatment and EGb pretreatment. Salicylate induced GluN2B upregulation in the cochlea was considered to be associated with tinnitus development [2]. Recently, increased level of GluN2B in the IC was suggested as potential pathological findings to elicit tinnitus [7]. In this perspective, EGb pretreatment has been implicated to decrease GluN2B induced excitotoxicity, which may lead to ameliorate tinnitus development. Although our findings are significant, there are several study limitations that should be controlled in further investigations.

Firstly, although the present study was conducted on a salicylate-induced ototoxicity model with similar manner to previous 
studies, ototoxicity after SS injection was not guaranteed by OAE changes after SS. Moreover, we did not assess the correlation between GluN2B expression and OAE thresholds following EGb pretreatment. Based on our results, EGb pretreatment may prevent $\mathrm{OHC}$ damage and subsequently maintain OAE responses to some extent. Additional information on the precise association between OAE responses and GluN2B expression from a larger sample size is crucial. Secondly, we only evaluated the neuroprotective effect of EGb through a cross-sectional study. Precise interpretation of the GluN2B expression changes with regard to synaptic plasticity, might be hindered in our study and should thus be evaluated in future studies. Thirdly, although the present study assessed the therapeutic implications of EGb pretreatment on GluN2B expression, we also suggested a potential mechanism that might be associated with salicylate-induced ototoxicity, including $\mathrm{Na}^{+}$or $\mathrm{Ca}^{2+}$ overload and proinflammatory cytokine release $[8,23,24]$. Additional evidence from a larger sample size is necessary to address the precise molecular mechanisms, such as excitotoxicity, changes in neuronal apoptosis, behind EGb-mediated GluN2B expression. Increased GluN2B expression in the IC following SS injection was attenuated by the administration of EGb. Thus, EGb may play a role in GluN2B subunit regulation in the IC.

\section{CONFLICT OF INTEREST}

No potential conflict of interest relevant to this article was reported.

\section{ACKNOWLEDGMENTS}

This study was supported by a clinical research grant provided by Seoul National University Boramae Medical Center, Seoul, Republic of Korea.

\section{ORCID}

Sang-Yeon Lee https://orcid.org/0000-0003-3566-8708

Young Ho Kim https://orcid.org/0000-0001-7328-5068

\section{REFERENCES}

1. Sinha GP, Sabri F, Dimitriadis EK, Iwasa KH. Organization of membrane motor in outer hair cells: an atomic force microscopic study. Pflugers Arch. 2010 Feb;459(3):427-39.

2. Guitton MJ, Caston J, Ruel J, Johnson RM, Pujol R, Puel JL. Salicylate induces tinnitus through activation of cochlear NMDA receptors. J Neurosci. 2003 May;23(9):3944-52.

3. Parsons MP, Raymond LA. Extrasynaptic NMDA receptor involve- ment in central nervous system disorders. Neuron. 2014 Apr;82(2): 279-93.

4. Oestreicher E,ArnoldW, Felix D. Neurotransmission of the cochlear inner hair cell synapse-implications for inner ear therapy. In: Felix D, Oestreicher E, editors. Rational pharmacotherapy of the inner ear. Basel: Karger Publishers; 2002. p. 131-9.

5. ImsuwansriT, Hoare DJ, PhaisaltuntiwongsW, Srisubat A, Snidvongs K. Glutamate receptor antagonists for tinnitus. Cochrane Database Syst Rev. 2016 Oct;10:CD012391.

6. Mitchell JA, Akarasereenont P,Thiemermann C, Flower RJ, Vane JR. Selectivity of nonsteroidal antiinflammatory drugs as inhibitors of constitutive and inducible cyclooxygenase. Proc Natl Acad Sci U SA. 1993 Dec;90(24):11693-7.

7. Hu SS, Mei L, Chen JY, Huang ZW, Wu H. Expression of immediateearly genes in the inferior colliculus and auditory cortex in salicylateinduced tinnitus in rat. Eur J Histochem. 2014 Mar;58(1):2294.

8. Szasz BK, Lenkey N, Barth AM, Mike A, Somogyvari Z, Farkas O, et al. Converging effects of Ginkgo biloba extract at the level of transmitter release, NMDA and sodium currents and dendritic spikes. Planta Med. 2008 Aug;74(10):1235-9.

9. Dave JR, Williams AJ, Moffett JR, Koenig ML, Tortella FC. Studies on neuronal apoptosis in primary forebrain cultures: neuroprotective/anti-apoptotic action of NR2B NMDA antagonists. Neurotox Res. 2003 Jan;5(4):255-64.

10. Ahlemeyer B, Krieglstein J. Pharmacological studies supporting the therapeutic use of Ginkgo biloba extract for Alzheimer's disease. Pharmacopsychiatry. 2003 Jun;36 Suppl 1:S8-14.

11. Li S, Luo J,Wang X, Guan BC, Sun CK. Effects of Ginkgo biloba extracts on NMDA-activated currents in acutely isolated hippocampal neurons of the rat. Phytother Res. 2011 Jan;25(1):137-41.

12. National Research Council of the National Academies. Guide for the care and use of laboratory animals. Washington (DC): National Academies Press; 2010.

13. Sheppard A, Hayes SH, Chen GD, Ralli M, Salvi R. Review of salicylate-induced hearing loss, neurotoxicity, tinnitus and neuropathophysiology. Acta Otorhinolaryngol Ital. 2014 Apr;34(2):79-93.

14. Im GJ, Choi J, Chang JW, Kim SJ, Kim HI, Jung HH. Expression of insulin-like growth factors in a mouse model of salicylate ototoxicity. Clin Exp Otorhinolaryngol. 2010 Sep;3(3):115-21.

15. Choi IS, Sung JY, Lee KS, Chang CS, Jun BH. Effects of salicylate on $\mathrm{ABR}$ and ECoG in guinea pig. Korean J Otolaryngol Head Neck Surg. 1999 Mar;42(3):290-7.

16. Fioretti A, Eibenstein A, Fusetti M. New trends in tinnitus management. Open Neurol J. 2011 Mar;5:12-7.

17. Hwang JH, Chen JC, Yang SY, Wang MF, Liu TC, Chan YC. Expression of COX-2 and NMDA receptor genes at the cochlea and midbrain in salicylate-induced tinnitus. Laryngoscope. 2011 Feb;121(2): 361-4.

18. Hu SS, Mei L, Chen JY, Huang ZW,Wu H. Expression of immediateearly genes in the dorsal cochlear nucleus in salicylate-induced tinnitus. Eur Arch Otorhinolaryngol. 2016 Feb;273(2):325-32.

19. Yashiro K, Philpot BD. Regulation of NMDA receptor subunit expression and its implications for LTD, LTP, and metaplasticity. Neuropharmacology. 2008 Dec;55(7):1081-94.

20. Zhao J, Wang B, Wang X, Shang X. Up-regulation of $\mathrm{Ca}(2+) / \mathrm{CaM}$ KII/CREB signaling in salicylate-induced tinnitus in rats. Mol Cell Biochem. 2018 Nov;448(1-2):71-6.

21. Huang DS, Lin HY, Lee-Chen GJ, Hsieh-Li HM, Wu CH, Lin JY.Treatment with a Ginkgo biloba extract, EGb 761, inhibits excitotoxicity in an animal model of spinocerebellar ataxia type 17. Drug Des DevelTher. 2016 Feb;10:723-31.

22. Wheeler D, Knapp E, Bandaru VV, Wang Y, Knorr D, Poirier C, et al. Tumor necrosis factor-alpha-induced neutral sphingomyelinase-2 modulates synaptic plasticity by controlling the membrane insertion 
of NMDA receptors. J Neurochem. 2009 Jun;109(5):1237-49.

23. Hwang JH, Chen JC, Chan YC. Effects of C-phycocyanin and Spirulina on salicylate-induced tinnitus, expression of NMDA receptor and inflammatory genes. PLoS One. 2013;8(3):e58215.
24. Kaur S, Sharma N, Nehru B. Anti-inflammatory effects of Ginkgo biloba extract against trimethyltin-induced hippocampal neuronal injury. Inflammopharmacology. 2018 Feb;26(1):87-104. 